\title{
The use of complementary and alternative medicine in cancer patient of north India.
}

\author{
Professor Bhatt. Suman Singh, Dr. Rmlims, Vibhuti Khand \\ gomtinager, Lucknow, up.
}

Abstract: Background: The aim of this study was to explore the use of complementary and alternative medicine (CAM) among cancer patients undergoing treatment in north Indian region.

Key words-alternative medicine, complementary medicine, homeopathic.

\section{Introduction-}

Complementary and alternative medicine (CAM) is the term for medical products and practices that are not part of standard care. Standard care is what medical doctors, doctors of osteopathy and allied health professionals, such as registered nurses and physical therapists, practice. Alternative medicine means treatments that you use instead of standard ones. Complementary medicine means nonstandard treatments that you use along with standard one. People want simple information to be made available about cost, effectiveness and feeling of well being.

\section{Objectives-}

To examine pattern of alternative medicine, economical co relation and benefits of CAM.

\section{Methods:}

A descriptive survey design was developed. Data was collected through a descriptive questionnaire from 84patients visiting OPD.The aspect like period of CAM, money spended, type of therapies used and benefits of medicine and feeling of wellness among patient was assessed/observed through direct interview. Any disagreement was settled through discussion

III. Results:

Use of alternative medicine along with allopathic

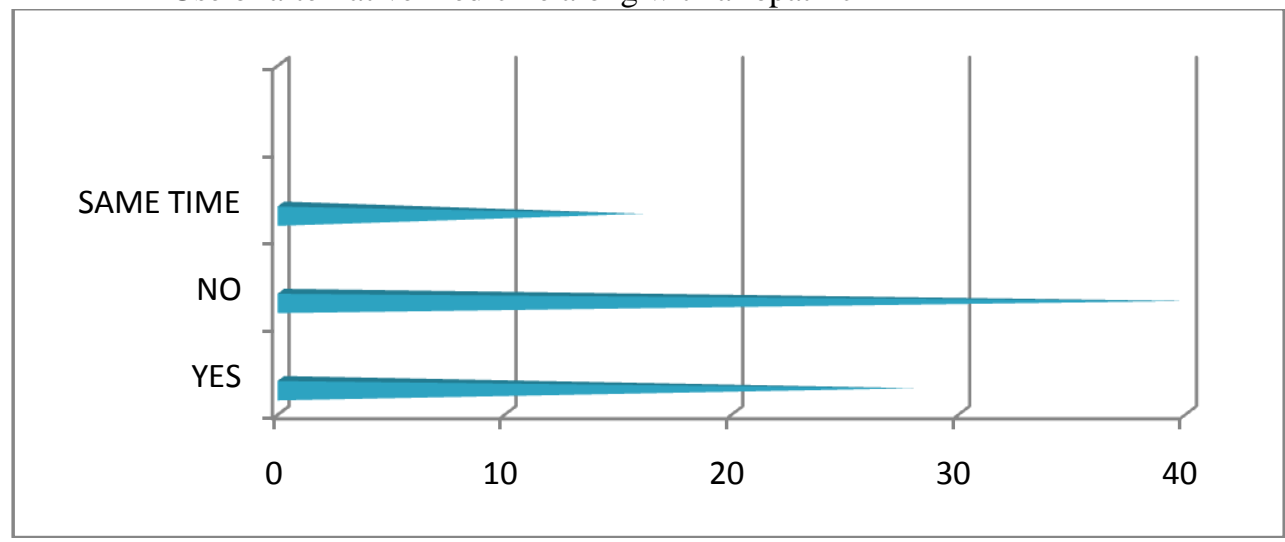

2-Referal towards CAM- 

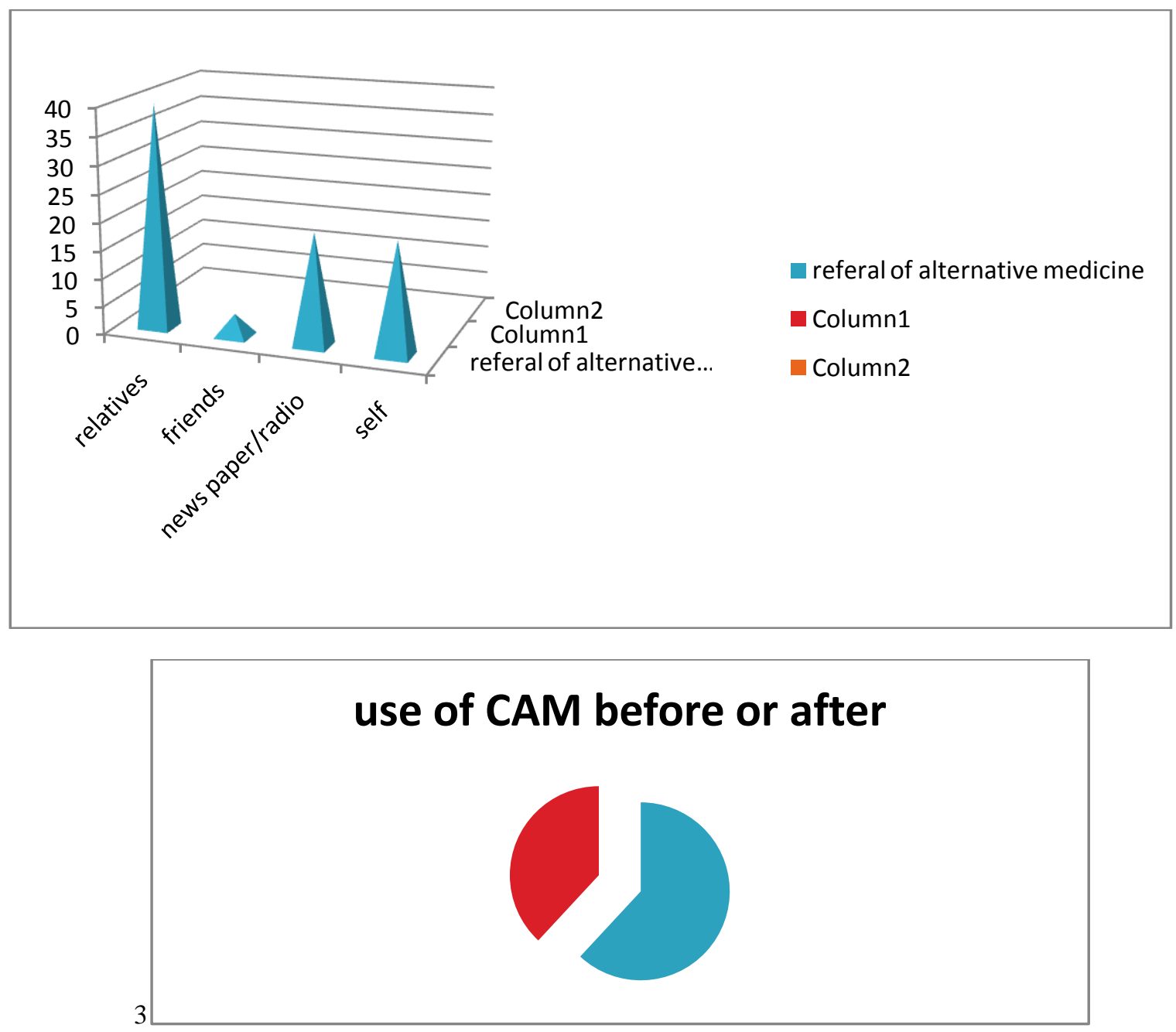

More than $60 \%$ patients uses CAM after biopsy also.

4- time period of medicine use.

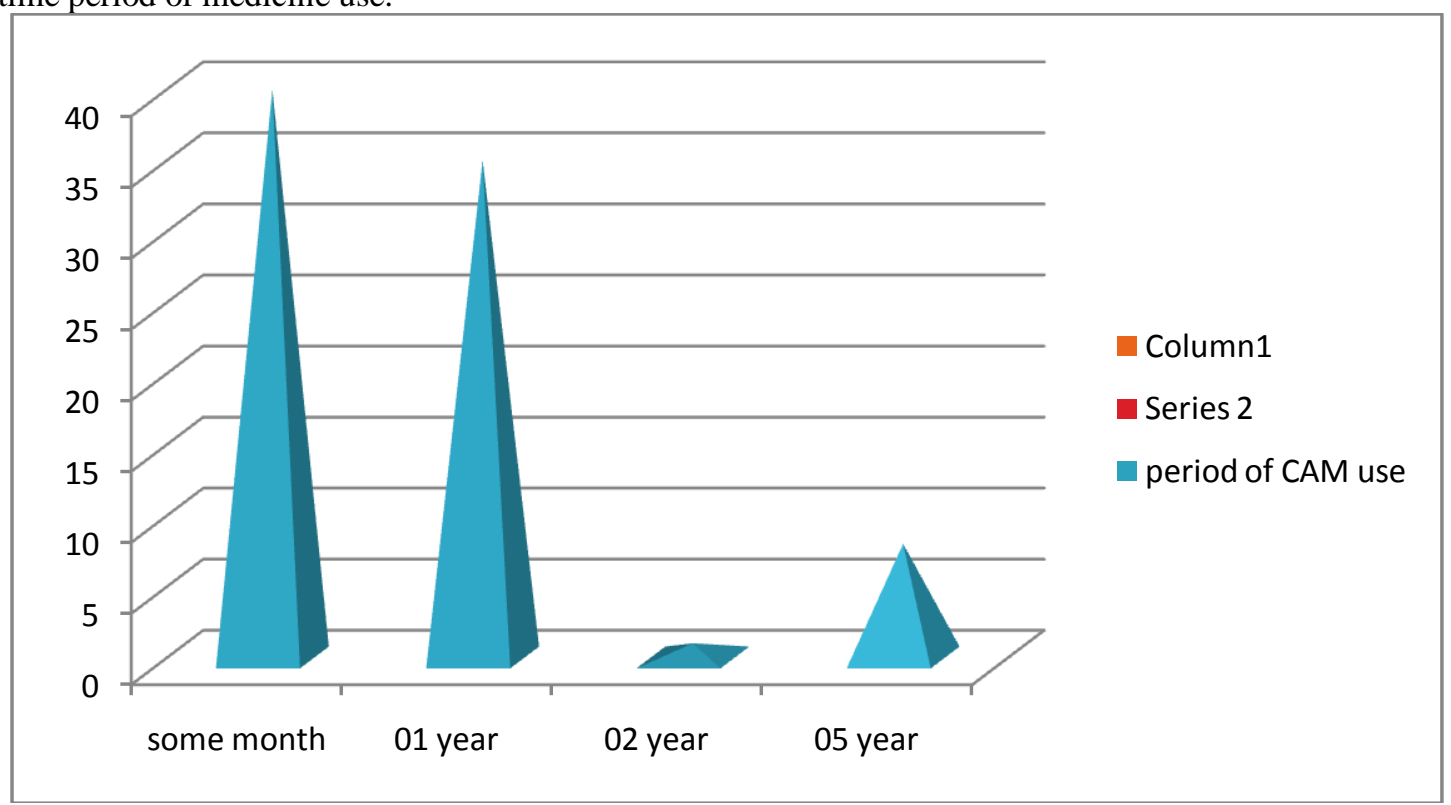


5- Money spended by the patient.

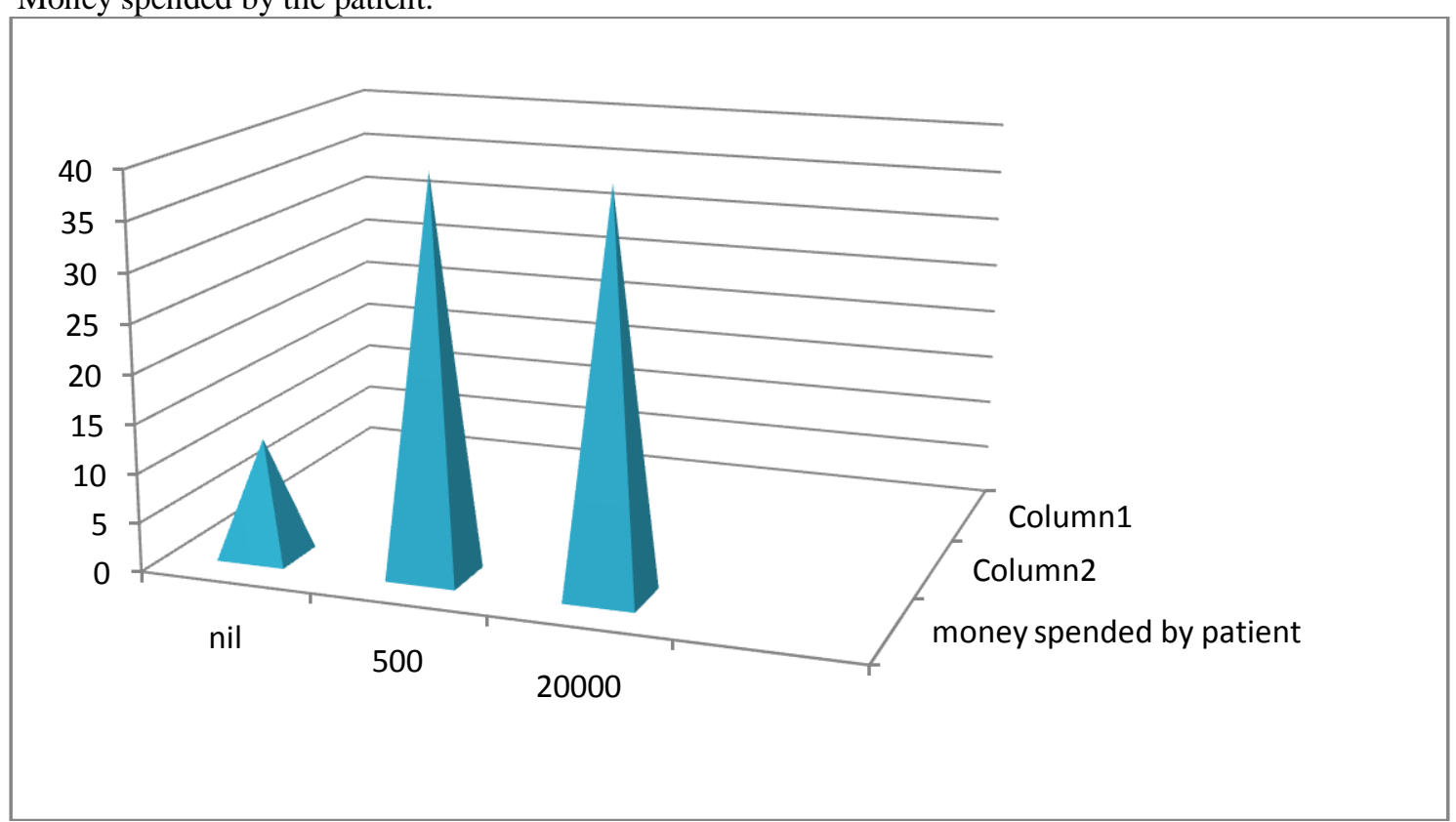

-6 Regularity in use of CAM.

\section{REGULARITY}

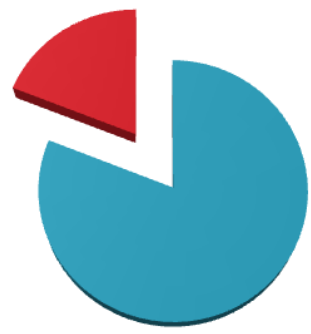

Meditation, massage, homeopathy, yoga, naturopathy were most common.

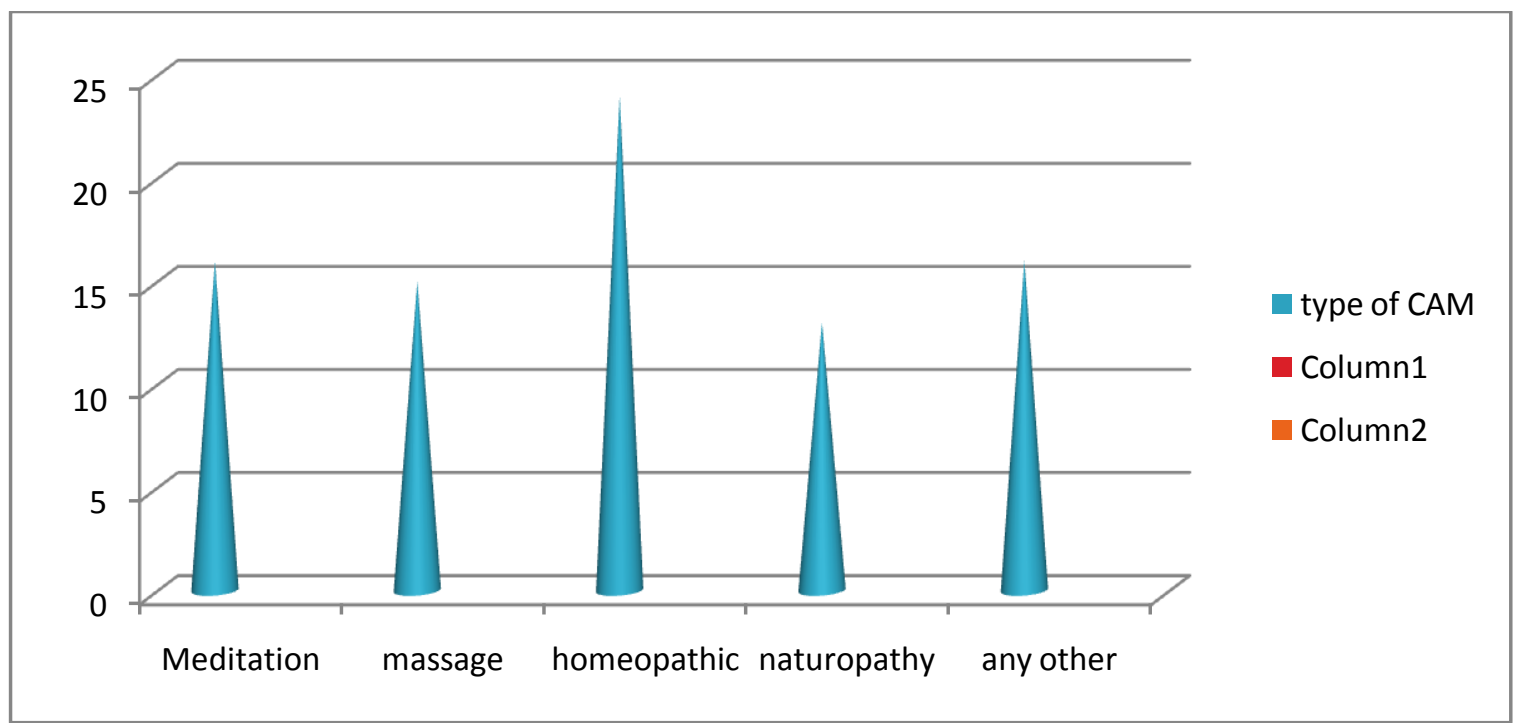

Benefits of alternative medicine. 


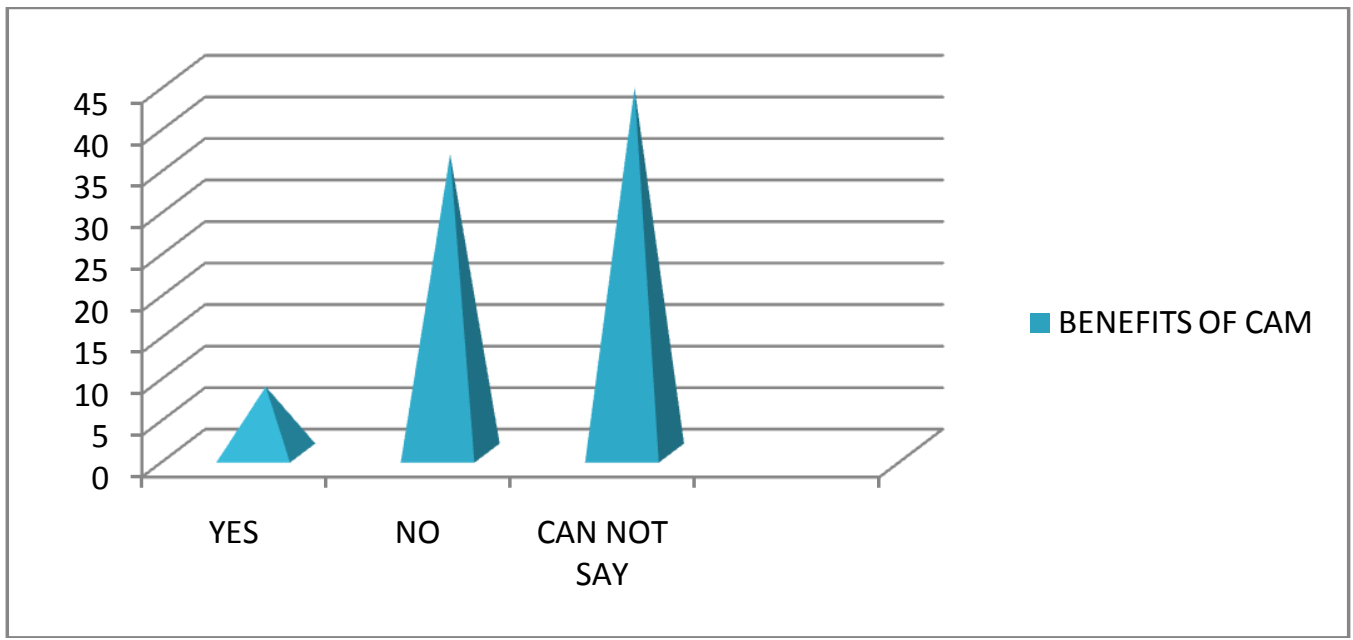

Feeling of well being

\section{feeling of well being}

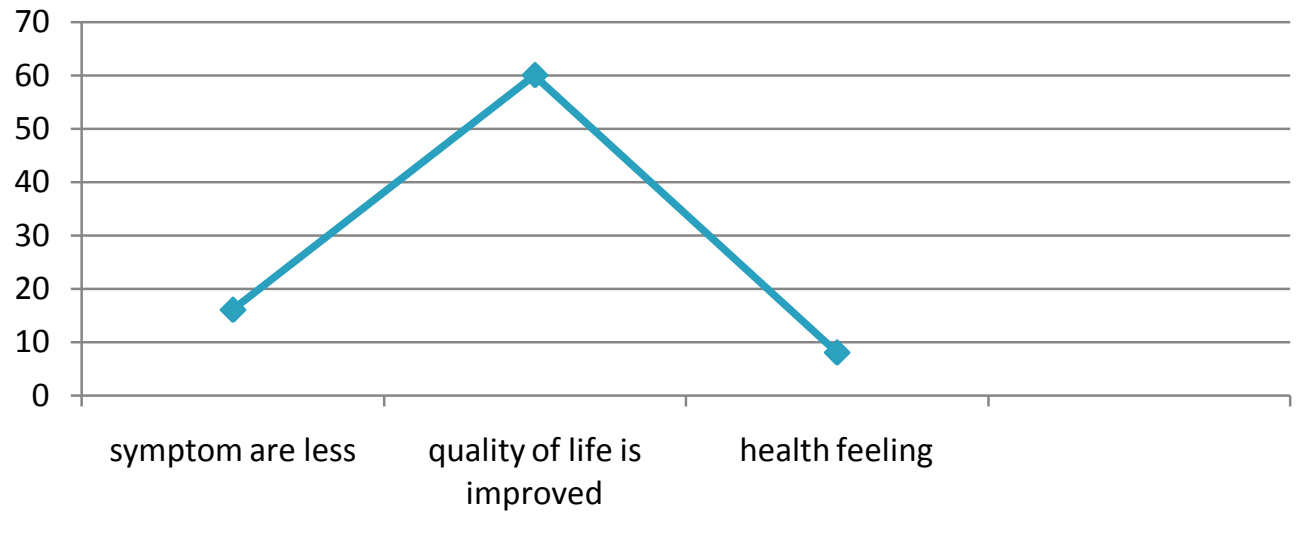

Data suggest that CAM is popular among cancer patients 50\% using some form of CAM with treatment .reference of CAM was found self or relatives. In 50\% cases remedies were used from years together in $40 \%$ cases CAM was used Before biopsy and 60\% cases it continued after biopsy also.CAM is not practiced free of cost more than 20000rs were spended by $60 \%$ of patient $.70 \%$ patient were regular in use of CAM. Most commonly used together with homeopathy, Massage ,mediation and naturopathy .only $17 \%$ patients felt benefited from CAM and and $41 \%$ were not feeling benefited $42 \%$ were could not say anything. Multivariate analysis suggested that the profile of the CAM user was that of younger people, female and with higher educational level. The source of information was mainly from friends/family and the media, while physicians and nurses played a small part in providing CAM-related information. 99\% patient felt sick after use of CAM.

\section{Conclusions:}

It is imperative that health professionals explore the use of CAM with their cancer patients the use of CAM is not able to eradicate feeling of sickness but helps in managing symptoms of cancer and provides improvisation of feeling of wellbeing only after paying cost of CAM in north India. 